\title{
Manejo Multidisciplinario de la Cavidad Oral en Pacientes COVID-19 bajo Ventilación Mecánica Invasiva. Rol del Equipo Odontológico
}

\author{
Multidisciplinary Management of the Oral Cavity in COVID-19 Patients \\ under Invasive Mechanical Ventilation. Role of the Dental Team
}

Humeres Sigala Carlos; Márquez Agustín²; González Ritchie Paulina; Valenzuela Ramos Ricardo4; Rivera Saavedra Marcelo5; Vásquez Pablo ${ }^{6}$ \& Araya Salas Cristóbal ${ }^{7}$

\begin{abstract}
HUMERES, S. C.; MÁRQUEZ, A.; GONZÁLEZ, R. P.; VALENZUELA, R. R.; RIVERA, S. M.; VÁSQUEZ, P. \& A. S. C. Manejo multidisciplinario de la cavidad oral en pacientes COVID-19 bajo ventilación mecánica invasiva. Rol del equipo odontológico. Int. J. Odontostomat., 14(4):701-704, 2020.
\end{abstract}

RESUMEN: El objetivo de este artículo es presentar algunas consideraciones odontológicas y un protocolo de mantención para el manejo y cuidado de cavidad oral en pacientes en ventilación mecánica invasiva (VMI) en unidades de cuidados intensivos hospitalarios. Además, presentar recomendaciones para la implementación de estas. Desde el desarrollo del COVID-19 en el mundo, los casos de VMI prolongada han aumentado y junto con ellos, se ha visibilizado las complicaciones propias de este tipo de terapia, dentro de las cuales encontramos aquellas que afectan la cavidad oral. Los centros hospitalarios normalmente cuentan con servicios dentales conformados por distintas especialidades, las cuales en un trabajo mancomunado pueden entregar herramientas y educar al personal encargado para el cuidado de la cavidad oral en estos pacientes si es que son requeridos, siendo el odontólogo el encargado de evaluar las distintas situaciones clínicas y entregar directrices para la prevención y tratamiento de patología de la cavidad oral. En este trabajo se describen los pasos a seguir para realizar una correcta higiene oral de estos pacientes y el manejo de las distintas complicaciones posibles de observar, junto con la experiencia local de cómo solucionarlos de la mejor manera. Consideramos importante que las unidades de cuidados intensivos ( $\mathrm{UCl}$ ) conozcan el rol del odontólogo y las acciones que este equipo puede aportar en el cuidado de los pacientes hospitalizados para disminuir los riesgos asociados a ventilación prolongada, basados principalmente en el manejo de la higiene oral y lesiones asociadas.

PALABRAS CLAVE: COVID-19, Odontología, Ventilación mecánica invasiva, Equipo Dental.

\section{INTRODUCCIÓN}

La pandemia por la enfermedad del coronavirus (COVID-19), causada por el virus SARS-CoV-2, originada en Wuhan, China, se ha convertido en un problema mayor de salud pública no solo en China, sino también a través de todo el mundo (Araya-Salas, 2020; Meng et al., 2020). Este virus, ha demostrado una clara relación con el SARS-CoV-1 (agente del SARS); por lo tanto, se indica a los murciélagos como un potencial origen con el reservorio. Debido a lo anterior, este virus emerge como una zoonosis a consecuencia de la trasgresión de la barrera específica. Tiene una letalidad de $1,38 \%$, lo que si bien no es tan alto comparado con otras enfermedades, tiene la gran problemática que causa una gran cantidad de casos en un corto periódo de tiempo, donde los casos más graves necesitarán ser internados en unidades de cuidados intensivos (UCl) (Canals, 2020; Faria et al., 2020).

\footnotetext{
${ }^{1}$ Especialista en Patología Buco Máxilofacial, Hospital Regional de Antofagasta Dr. Leonardo Guzmán, Antofagasta, Chile.

${ }^{2}$ Magister en Patología Buco Máxilofacial, Staff Equipo Cabeza y Cuello, Instituto Nacional del Cáncer, Chile.

${ }^{3}$ Especialista en Cirugía BucoMáxilofacial, Hospital Regional de Antofagasta Dr. Leonardo Guzmán, Antofagasta, Chile.

${ }^{4}$ Especialista en Periodoncia, Hospital Regional de Antofagasta Dr. Leonardo Guzmán, Antofagasta, Chile.

5. Especialista en Ortodoncia, Hospital Regional de Antofagasta Dr. Leonardo Guzmán, Antofagasta, Chile.

6. Fonoaudiólogo, Equipo Cabeza y Cuello, Instituto Nacional del Cáncer, Chile.

${ }^{7}$ Especialista en Patología Buco Máxilofacial, Staff Equipo Cabeza y Cuello, Instituto Nacional del Cáncer, Chile.
} 
Según información de estudios prospectivos internacionales, el promedio de edad de los pacientes hospitalizados por infección de COVID-19 es de 62 años, donde aproximadamente un $22 \%$ presentará un estado crítico. En el transcurso de esta enfermedad severa, entre un $80 \%-88 \%$ de los pacientes críticos requerirá ventilación mecánica invasiva (VMI) en algún momento, con un promedio de aproximadamente 18 días de uso (IQR 9-28). Los pacientes que sobreviven, tiene una media de uso de VMI de 27 días, mientras aquellos que mueren tienen un tiempo de uso promedio de 10 días (Cumming et al, 2020).

La VMI se entiende como la entrega de presión positiva de forma orotraqueal o por traqueostomía, usada para reemplazar total o parcialmente la función respiratoria espontánea al realizar el trabajo respiratorio, permitiendo el intercambio de gases en aquellos pacientes con falla respiratoria. Su aplicación se divide basicamente en las fases de iniciación, mantención y retiro (weaning) (Slutsky, 1993).

\section{Consideraciones Generales}

Los pacientes en VMI presentan una gran disminución de la capacidad ventilatoria autónoma en el tiempo, además, se genera la pérdida de deglución y reflejo de tos por el estado de sedación e impedimento físico, lo que determina la acumulación de todas las secreciones del tracto aéreo superior fluyendo por gravedad a lugares donde se puedan asentar. Estos fluídos ricos en mucina, son de gran adherencia a las mucosas, pero debido a la carencia de deglución se conforma el medio ideal para que microorganismos oportunistas, saprófitos y nosocomiales presenten un aumento en su crecimiento. Este fluido, de acumularse también podrá ser aspirado de manera involuntaria, evento que puede significar el desarrollo de un síndrome de distrés respiratorio por aspiración o permitir la evolución a una neumonía asociada a ventilación, siendo esta última, una de las principales complicaciones graves de la ventilación prolongada (American Thoracic Society \& Infectious Diseases Society of America, 2005).

En la cavidad oral, el daño se ve aumentado a través de factores como la posición en prono que se debe adoptar en algunos pacientes en VMI para aumentar la perfusión pulmonar y comorbilidades de base como obesidad, donde la lengua se desplaza a una posición anterior, quedando en ocasiones bajo la presión del tubo (Cummings et al.). Además de lo mencionado, se puede apreciar el traumatismo de los dientes, sumado al factor gravitacional; todas estas situa- ciones pueden provocar complicaciones tales como: esfacelos mucosos, edema lingual severo, úlceras traumáticas y por presión, boca seca y resequedad de las mucosas.

\section{Manejo de complicaciones orales en VMI}

Para disminuir las posibles complicaciones que puedan poner en riesgo el estado de salud general del paciente en VMI, como la aspiración de fluidos, se debe establecer una rutina diaria de higiene oral diseñada para cada paciente dependiente de la fase de ventilación en la que se encuentre. Esta rutina debe permitir la disminución del rieso de complicaciones en cavidad oral, propios de la VMI.

\section{Materiales:}

- Cánula de aspiración

- Jeringa irrigación

- Sistema de aspiración

- Copela $20 \mathrm{ml}$.

- Bandeja de curación (con pinza quirúrgica)

- Clorhexidina (CHX) 0,12\%

- Peróxido de hidrogeno 1 \% (Checchi et al., 2020)

- Gasa

- Compresas

- Silicona por adición

- Dispositivo intraoral individual (abre boca individualizado, estándar o tornillo de disyunción maxilar tipo Hyrax modificado e individualizado con silicona por adición)

- Gel humectante de mucosas (Sucralfato)

- Lámpara Luz Led de Baja Potencia (Laser Therapy $\mathrm{DMC})$

Paciente en ventilación orotraqueal:

Condición: Sedado. Posición: Decubito supino, semi fowler.

a) Armar jeringa con $\mathrm{CHX} 0,12 \%$ o Peróxido de $\mathrm{Hi}$ drógeno $1 \%$.

b) Limpiar con irrigación y gasa de manera suave toda la mucosa oral (labio, geniana, piso de cavidad oral, encías, paladar y al final lengua).

c) Eliminar de manera activa con pinza quirúrgica esfacelos mucosos en cavidad oral (saliva, sangre coagulada).

d) Evitar manejo de tubo orotraqueal y correcto manejo de aspiración de fluidos.

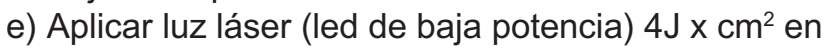
lesiones ulceradas o erosionadas. 
f) Modelar silicona por adición en dientes que representen zonas potenciales de trauma o impacto de dientes en la mucosa oral (Fig. 1).

g) Instalación de dispositivo intraoral para mantener una adecuada apertura bucal y correcta posibilidad de aseo. Debe ser individualizado con silicona por adición para disminuir el riesgo de desalojo y amarrado con compresa o cinta de seguridad para evitar aspiración accidental. (Fig. 2).

h) Aplicación de gel humectante (Sucralfato o similares).

i) Entregar indicaciones de higiene básica oral (pasos a, b y c) cada 8 horas y recomendaciones a personal encargado de higiene y mantención oral del paciente.

j) Control cada $24 \mathrm{hrs}-48 \mathrm{hrs}$ por odontólogo especialista.

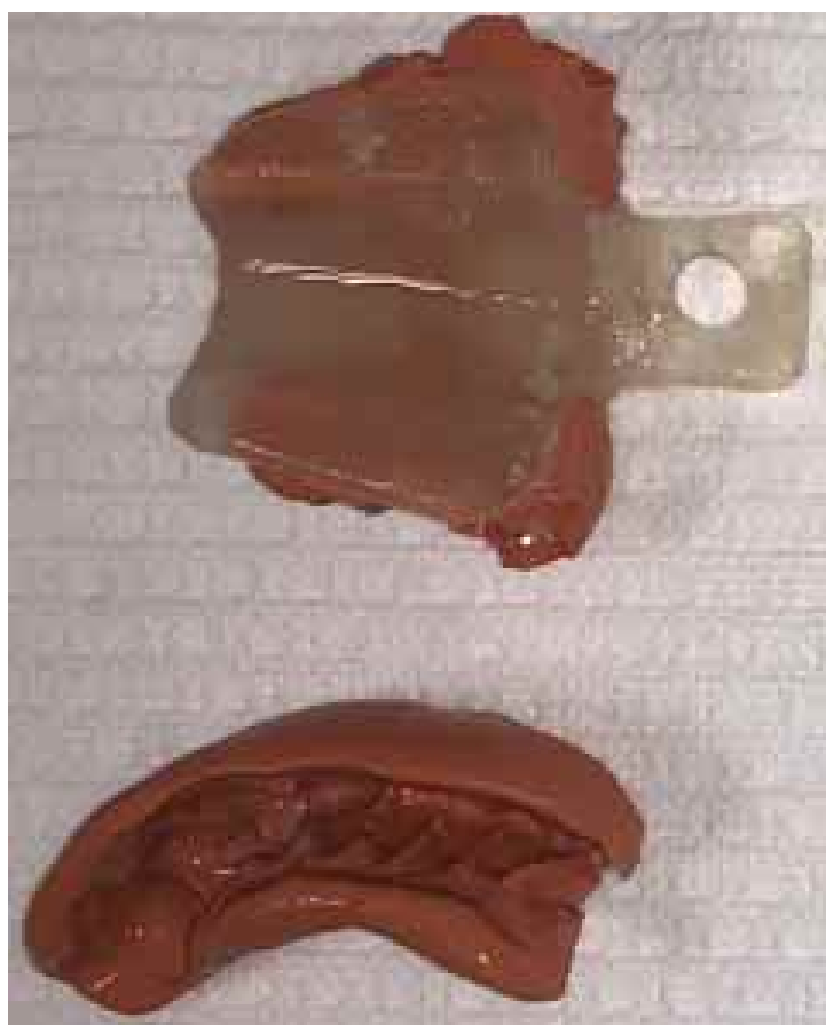

Fig. 1. Superior: Dispositivo intraoral individualizado con silicona por adición. Inferior: Descanso lingual individualizado para evitar ulceraciones en cara ventral de lengua.

\section{Recomendaciones}

- El uso de enjuagues de peróxido de hidrógeno $1 \%$ se recomienda como antiséptico eficaz de disminuir la carga viral de SARS-COV2 en cavidad oral.

- Siempre previo a realizar el procedimiento descrito, chequear con equipo a cargo la condición ventilatoria óptima para disminuir riesgos procedimentales.

- Extremar cuidados de aspiración con sistemas de eyección y contención de excesos de líquido con gasas.

Nota: Pacientes en posición prono solamente se debe evaluar posición lingual no traumática a la espera de llevar a posición supina.

\section{CONCLUSIONES}

Durante la terapia de VMI, es esperable que el paciente pueda experimentar algunas complicaciones inherentes a este procedimiento. Estas complicaciones se presentan de una forma aún más severa en pacientes que experimentan tiempos prologandos de $\mathrm{VMI}$ en unidades intensivas (> 18 días), posición prono y obesidad, por lo que es recomendable el control en el tiempo por parte de un equipo odontológico capacitado para dar una solución adecuada y oportuna, integrándose al equipo de salud que también participa en la mantención del sistema estomatognático, como kinesiólogos, fonoaudiólogos y enfermeras, brindando el soporte necesario específico a cada paciente.

Creemos firmemente que el rol del equipo odontológico en los pacientes que presenten complicaciones asociadas a la VMI de larga estadía (principalmente asociada a infección por COVID19) es fundamental tanto para el manejo de estas, como para prestar asesoría al equipo intensivista, ya que un manejo integral de la cavidad oral permitirá al paciente presentar un mejor outcome durante su VMI, y al finalizar esta e iniciar su fase de rehabilita-

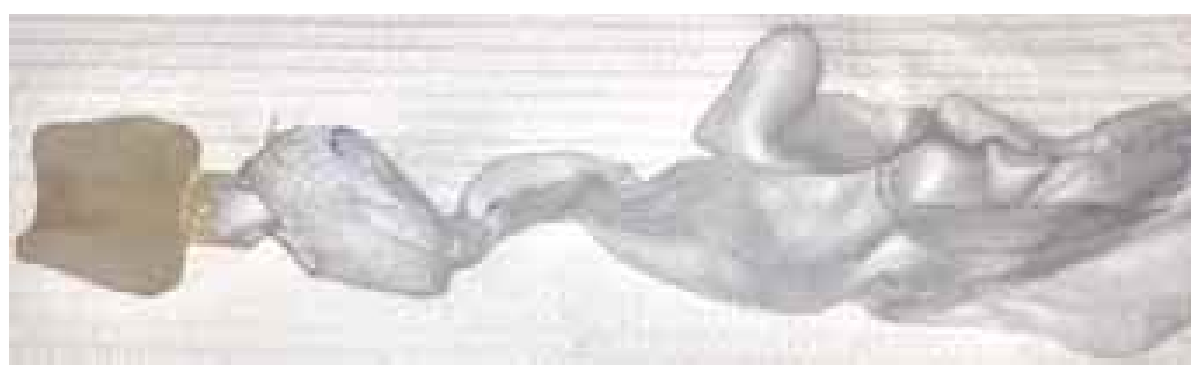

Fig. 2. Dispositivo intraoral (abreboca) con amarre de seguridad. 
ción al permitir un correcto posicionamiento de la lengua debido a la ayuda de los dispositivos intraorales. Lo anterior, permitirá la disminución del edema lingual, evitando el trauma a repetición que pudiera significar un sangrado activo en cavidad oral con posibilidad de desencadenar eventos aspirativos que pongan en riesgo el estado del paciente. Finalmente, queremos recordar y hacer énfasis, que es el odontólogo el profesional del equipo de salud especializado en el diagnóstico, tratamiento y prevención de las patologías en cavidad oral, por lo tanto, es el profesional llamado a atender y resolver estos requerimientos si es que el equipo médico así lo indica, ya que la oportuna intervención del equipo odontológico repercutirá en la prevención y control de eventos de riesgo, permitiendo un mejor manejo de las complicaciones asociadas y contribuirá a una eficaz recuperación general de estos pacientes.

HUMERES, S. C.; MÁRQUEZ, A.; GONZÁLEZ, R. P.; VALENZUELA, R. R.; RIVERA, S. M.; VÁSQUEZ, P. \& A. S. C. Multidisciplinary management of the oral cavity in COVID-19 Patients under invasive mechanical ventilation. Role of the dentalteam. Int. J. Odontostomat., 14(4):701-704, 2020.

ABSTRACT: The aim of this sutudy dental considerations and an oral cavity maintenance and care protocol in patients on invasive mechanical ventilation (IMV) in hospital intensive care units. Since the development of COVID-19 in the world, cases of prolonged IMV have increased and together with them, the complications of this type of therapy have been made visible, among which we find those that affect the oral cavity. Hospital centers normally have dental services made up of different specialties, which in a joint effort can provide tools and educate the personnel in charge of the care of the oral cavity in these patients if they are required, being the dentist the one in charge of evaluating and deliver guidelines for the prevention and treatment of pathology of the oral cavity. This work describes the steps to be followed to carry out correct oral hygiene in these patients and the management of the different complications that may be observed, along with local experience on how to best solve them. We consider important that the intensive care units (ICU) get to know the role of the dentist in the hospital environment and that they become part of the care of hospitalized patients to reduce the risks associated with prolonged ventilation, based mainly on oral hygiene management and associated injuries.

KEY WORDS: COVID-19, Odontology, Invasive Mechanical Ventilation, Dental team.

\section{REFERENCIAS BIBLIOGRÁFICAS}

American Thoracic Society \& Infectious Diseases Society of America. Guidelines for the management of adults with hospital-acquired, ventilator-associated, and healthcare-associated pneumonia. Am. J. Respir. Crit. Care Med., 171(4):388-416, 2005.

Araya-Salas, C. Considerations for emergency dental care and preventive measures for COVID-19 (SARS-CoV 2). Int. J. Odontostomat., 14(3):268-70, 2020.

Canals, L. M. Conceptos para una buena toma de decisiones en la pandemia COVID-19 en Chile. Rev. Chil. Infectol., 37(2):170-2, 2020.

Checchi, V.; Bellini, P.; Bencivenni, D. \& Consolo, U. COVID-19 dentistry-related aspects: a literature overview. Int. Dent. J., 2020. DOI: https://www.doi.org/10.1111/idj.12601

Cummings, M. J.; Baldwin, M. R.; Abrams, D.; Jacobson, S. D.; Meyer, B. J.; Balough, E. M.; Aaron, J. G.; Claassen, J.; Rabbani, L. E.; Hastie, J.; et al. Epidemiology, clinical course, and outcomes of critically ill adults with COVID-19 in New York City: a prospective cohort study. Lancet, 395(10239):1763-70, 2020.

Faria, K. M.; Gomes-Silva, W.; Kauark-Fontes, E.; Bonfim-Alves, C. G.; Kowalski, L. P.; Prado-Ribeiro, A. C.; Vechiato-Filho, A. J.; Lopes, M. A.; Marta, G. N.; de Castro Jr., G.; et al. Impact of pandemic COVID-19 outbreak on oral mucositis preventive and treatment protocols: new perspectives for extraoral photobiomodulation therapy. Support. Care Cancer, 2020. DOI: https://www.doi.org/10.1007/s00520-020-05636-1

Meng, L.; Hua, F. \& Bian, Z. Coronavirus Disease 2019 (COVID19): emerging and future challenges for dental and oral medicine. J. Dent. Res., 99(5):481-7, 2020.

Slutsky, A. S. Mechanical ventilation. American College of Chest Physicians' Consensus Conference. Chest, 104(6):1833-59, 1993.

Autor de correspondencia:

Carlos Humeres Sigala

Hospital Regional Antofagasta

Azapa 5395. Edificio CDT

Unidad de Anatomía Patológica

Antofagasta

CHILE

Email:chumeres@gmail.com

Recibido : 11-8-2020

Aceptado:17-8-2020 